\title{
Mesothelin/CD3e Tri-specific T-cell Activating Construct HPN536
}

National Cancer Institute

\section{Source}

National Cancer Institute. Mesothelin/CD3e Tri-specific T-cell Activating Construct

HPN536. NCI Thesaurus. Code C162935.

recombinant antibody derivative composed of a tri-specific $\mathrm{T}$-cell activating construct ( $T$ riT AC) directed against the human tumor-associated antigen (TAA) mesothelin (MSLN) and the epsilon domain of $C D 3$ antigen (CD3e) found on T-lymphocytes which are both linked to either side of an anti-albumin sing le domain antibody, with potential immunostimulating and antineoplastic activities. Upon administration of the MSLN/CD3e TriT AC HPN536, the anti-MSLN single antibody domain targets and binds MSLN on tumor cells and the anti-CD3e single-chain variable fragment (scFv) targets and binds to CD3e on cytotoxic T-lymphocytes (CT Ls), thereby bringing MSLN-expressing tumor cells and CT Ls together, which results in the CT L-mediated cell death of MSLN-expressing tumor cells. The albumin-binding domain targets and binds to serum albumin, thereby extending the serum half-life of HPN536. MSLN is overexpressed on the surface of certain tumor cell types. Overexpression of MSLN is often associated with poor prognosis. 